Published in final edited form as:

Liver Transpl. 2011 December ; 17(12): 1443-1447. doi:10.1002/lt.22425.

\title{
Organ quality and quality of life after liver transplantation
}

\author{
Michael L. Volk ${ }^{1}$ and Michael Hagan ${ }^{2}$ \\ ${ }^{1}$ Division of Gastroenterology and Hepatology, University of Michigan \\ ${ }^{2} \mathrm{Gift}$ of Life Michigan
}

\begin{abstract}
Not only is there a limited supply of organs for liver transplantation, but the quality of available organs is not uniform. Risk factors such as donor age and cause of death are known to predict graft failure, but their impact on recipient quality of life has not been reported. We sent a quality of life survey to 299 adults at our institution who had received a liver transplant between 1-7 years prior to the study. Among the 171(57\%) who completed the Medical Outcomes Study Short-Form 36 (SF-36), their mean Physical and Mental Composite scores (PCS, MCS) were 61 and 66 respectively, with the highest scores in the Social Functioning subscale and the lowest scores in the Role Functioning/Physical and Energy/Fatigue subscales. The mean Donor Risk Index (DRI) of organs that subjects had received was 1.4(range 0.8-2.4). There was no correlation between SF-36 scores and DRI ( -4.8 and -2.8 change in PCS and MCS per unit increase in DRI, $p=0.43$ ), despite controlling for potential confounders including age, gender, hospitalization prior to transplant, MELD at transplant, years since transplant, prior transplant, and Charlson Comorbidity Index. In conclusion, we found no association between organ quality and quality of life after liver transplantation. If confirmed in prospective multi-center studies, this finding will be useful in counseling patients about whether or not to accept high risk organ offers.
\end{abstract}

\section{Keywords}

Extended criteria donors; donor risk index; graft failure

\section{Introduction}

Although liver disease ranks twelfth in overall causes of death in the United States, the true impact on society is likely much larger than this since people are affected in their prime of life. In fact, liver disease ranks fourth in overall causes of death among people age 45-54 ${ }^{1}$. Furthermore, end-stage liver disease leads to dysfunction in virtually every organ system, resulting in a very poor quality of life for those affected ${ }^{2}$. Liver transplantation has become the best treatment for most patients with end-stage liver disease; it prolongs survival ${ }^{3}$ and improves quality of life ${ }^{2}$. One of the main limitations continues to be the limited supply of organs.

Organ supply is not fixed, but rather depends on multiple factors including the quality of organs that are accepted for use. Various donor factors are known to be associated with the risk of graft failure after transplant, including donor age, anticipated ischemia time, and donation after cardiac death ${ }^{4}$. Each time an organ offer is made, the physician and patient

Corresponding author: Michael L Volk, MD, MSc, Division of Gastroenterology and Hepatology, University of Michigan Health System, 300 N Ingalls St, 7C27, Ann Arbor, MI 48109, (T) 734-232-1071, (F) 734-936-8944, mvolk@ med.umich.edu. 
must make the difficult decision whether to accept the organ being offered, or wait in hopes that a better one will come along.

One limitation in physicians' ability to counsel patients about this decision is the lack of information on how donor characteristics affect recipient quality of life (QOL), defined by the World Health Organization as "individuals' perceptions of their position in life in the context of the culture and value systems in which they live and in relation to their goals, expectations, standards, and concerns" 5 . In other words, if a patient accepts a higher risk organ and survives, will he or she experience the same QOL as recipients of an average quality liver? The answer to this question is particularly important since most high risk organs are transplanted into patients with low Model for End-stage Liver Disease (MELD) scores $^{6}$, who are predicted to derive little survival benefit from transplant ${ }^{7}$ and thus may be undergoing transplant predominately to improve their QOL. Therefore, the aim of this study was to determine whether organ quality is associated with post-transplant QOL among liver transplant recipients.

\section{Methods}

Subject population - a QOL survey was mailed to adults who underwent deceased donor liver transplantation between 1-7 years prior to the study. This time period was chosen because previous studies have shown that QOL after liver transplantation continues to rise until about 1 year post-transplant, at which time it plateaus ${ }^{2}$. Recipients of combined transplants were excluded. Clinical and demographic characteristics were obtained from the medical chart, and the Deyo adaptation of the Charlson Comorbidity Index was obtained from billing data ${ }^{8}$. Post-transplant variables were obtained by individual chart review, and included hospital length of stay, renal failure requiring dialysis after the transplant hospitalization, biliary complication requiring percutaneous transhepatic cholangiogram (PTC) tube, and biopsy-proved rejection. Informed consent was obtained for participation, and this study was approved by our Institutional Review Board.

Quality of life measurement - we used the Medical Outcomes Study Short Form-36 (SF-36) instrument because it has been previously used in liver transplant recipients and is more sensitive than other instruments to small changes ${ }^{9}$. The 36 items combine to form a Physical Composite Score (PCS) and Mental Composite Score (MCS) between 0-100, as well as eight subscales: physical functioning, role functioning/physical, role functioning/emotional, energy/fatigue, emotional well-being, social functioning, pain, and general health ${ }^{10}$. Scores are reported in raw scale units unless otherwise noted.

Organ quality - was measured using the Donor Risk Index (DRI) ${ }^{4}$, a continuous measure where each unit increase over 1 denotes the incremental risk of graft failure relative to "best quality" organs. The individual components of the DRI include donor age, race, height, cause of death, donation after cardiac death, split versus whole organ, regional or national share, and cold ischemia time. These data were obtained directly from our Organ Procurement Organization, with the exception of cold ischemia time which was obtained from our hospital's transplant database. Imputation was performed for 12/171 (7\%) of subjects with missing cold ischemia time, and an imputation flag was created to ensure that no bias was introduced by this method ${ }^{11}$. In cases where subjects had received multiple liver transplants, data from the most recent transplant was used.

Statistical analysis - bivariate comparisons were performed using t-test and Pearson's chisquare statistic for continuous and categorical data, respectively. Multivariable linear regression was used to determine variables independently associated with the raw scores of SF-36 PCS and MCS, as well as eight subscales. Although the SF-36 scores were not 
normally distributed, residual-versus-fitted plots demonstrated no evidence of heteroskedasticity, thus supporting the use of parametric methods. Power calculations showed that a sample size of 139 would be sufficient to detect a 10-unit decrease in SF-36 caused by DRI ( $10 \%$ of overall range of SF-36), assuming $\beta=0.8$ and $\alpha=0.05$. Calculations were performed using Stata v11.0 (Statacorp, College Station TX).

\section{Results}

The QOL survey was sent to 299 transplant recipients, and was completed by 171 (57\%). The respondents were less likely to be Hispanic ( $2 \%$ vs $11 \%, \mathrm{p}=0.03$ ), but otherwise did not differ from non-respondents by age, gender, race/ethnicity, primary diagnosis, or years since transplant. Demographic characteristics of the survey respondents can be seen in Table 1, in comparison to the entire population of adult liver transplant recipients at our center during the study period.

The mean Physical and Mental Composite SF-36 scores among the 171 respondents were 61 and 66 respectively, which is consistent with other studies of liver transplant recipients ${ }^{12}$. As shown in Table 2, when compared to the U.S. population, transplant recipients appear to have lower Physical Composite scores but similar Mental Composite scores ${ }^{13}$. Nineteen percent indicated that their quality of life had not improved since before their transplant, or had gotten worse.

The mean DRI among organs received by study subjects was 1.4 (standard deviation 0.4), which is consistent with organ quality in our transplant region ${ }^{11}$. By comparison, the mean DRI for the entire population of adult liver transplant recipients at our center during the study period was also 1.4. Rates of death and graft failure in the entire transplant population during the study period were $27 \%$ and $31 \%$ among recipients of organs with DRI $<=1.6$ $(\mathrm{n}=310)$, versus $36 \%$ and $38 \%$ among recipients of organs with DRI $>1.6(\mathrm{n}=154)(\mathrm{p}=0.04$ and 0.1 , respectively).

The distribution of DRI among survey respondents is shown in Figure 1. Subjects who received a transplant in more recent years tended to have higher DRI organs, as did those with lower lab MELD scores at transplant ( $\mathrm{p}<0.05$ for both comparisons). However, there was no association between DRI and post-transplant QOL, either by Physical or Mental Composite Scores or by each individual subscale. When organ quality was dichotomized into high risk (DRI >1.6) versus standard risk, the PCS and MCS were 61 and 66 in the standard risk group, and 60 and 66 in the high risk group ( $\mathrm{p}=\mathrm{NS}$ ). As shown in Table 3, this lack of association persisted even after controlling for multiple potential confounders including recipient age, gender, years since transplant, MELD at transplant, history of prior transplant, comorbidity index, or hospitalization immediately before transplant. Subjects who reported that their quality of life had not improved with transplant were distributed equally according to quality of organ they had received: $18 \%$ in the standard risk group and $24 \%$ in the high risk (DRI $>1.6)$ group $(\mathrm{p}=0.4)$.

Post-transplant complications are shown in Table 4, according to whether the subject received a standard risk organ or high risk organ. Among this study population who survived at least one year post-transplant, recipients of high risk organs had similar length of stay and were no more likely to require dialysis or PTC tube than recipients of standard risk organs. High risk organ recipients had lower rates of biopsy-proven rejection compared to recipients of standard risk organs $(\mathrm{p}=0.01)$. 


\section{Discussion}

This study found that organ quality, as measured by the Donor Risk Index, does not appear to affect post-transplant quality of life. The Physical Composite and Mental Composite scores of the SF-36 were virtually identical between subjects who received standard risk versus high risk (DRI $>1.6$ ) organs. This lack of clinically or statistically significant association persisted despite adjustment for multiple potential confounders such as age, medical comorbidities, and pre-transplant severity of liver disease.

It is important to reiterate that subjects in this study had to survive at least one year posttransplant to be included, and were on average 4 years out from transplant. Since DRI is known to be a strong predictor of graft failure within the first 1-3 years, the QOL implications of these findings are contingent upon the patient surviving these first years. This survival bias likely explains the lack of association between organ quality and posttransplant complications. Additionally, not all patients experienced improvements in QOL $19 \%$ felt they were no better or even worse off than before their transplant. Therefore, patients can be counseled as follows: if they accept a high-risk organ and do not die from graft failure, their quality of life may be no worse than if they had received a standard risk organ. Indeed, their quality of life would be much better than if they died on the waiting list, which might be the outcome for patients with high MELD scores who decline a high-risk organ.

This study is limited by the relatively small proportion of very high risk organs (DRI>2). Further studies are needed to determine whether such organs, which comprise less than $10 \%$ of liver transplants nationwide ${ }^{11}$, are associated with worse quality of life after transplantation. Second, the sample size was powered to detect a 10-unit change in the SF-36 score. An impact of organ quality on QOL of less than 10 units could be clinically significant, and could have been missed. Third, this was a single-center study. Variation in surgical technique at different centers could theoretically interact with organ quality and thus influence post-transplant quality of life via biliary or other complications. Furthermore, variation between centers in organ utilization and recipient/graft matching might limit the generalizability of these findings. A final limitation is the cross-sectional nature of this study, which may have been less sensitive for detecting minor changes in QOL than a longitudinal design, and also fails to account for pre-transplant QOL. It is possible that patients with worse pre-transplant QOL tended to receive better quality organs, even after controlling for variables such as MELD score.

In summary, organ quality does not appear to affect quality of life after liver transplantation, at least among low to moderate risk organs and among patients who survive at least one year after transplantation. If these findings are confirmed in multi-center prospective studies, they can be used to counsel patients when making difficult organ acceptance decisions.

\section{Abbreviations}
QOL Quality of Life
DRI Donor Risk Index

\section{Acknowledgments}

This work was funded by the American Gastroenterological Association (MLV) and by NIH K23DK085204 (MLV) 


\section{References}

1. Kung HC, Hoyert DL, Xu J, et al. Deaths: final data for 2005. Natl Vital Stat Rep. 2008; 56:1-120. [PubMed: 18512336]

2. Ratcliffe J, Longworth L, Young T, et al. Assessing health-related quality of life pre- and post-liver transplantation: a prospective multicenter study. Liver Transpl. 2002; 8:263-270. [PubMed: 11910572]

3. Merion RM, Schaubel DE, Dykstra DM, et al. The survival benefit of liver transplantation. Am J Transplant. 2005; 5:307-313. [PubMed: 15643990]

4. Feng S, Goodrich NP, Bragg-Gresham JL, et al. Characteristics associated with liver graft failure: the concept of a donor risk index. Am J Transplant. 2006; 6:783-790. [PubMed: 16539636]

5. Saab S, Ng V, Landaverde C, et al. Development of a disease-specific questionnaire to measure health-related quality of life in liver transplant recipients. Liver Transpl. 2011; 17:567-579. [PubMed: 21506245]

6. Volk ML, Lok AS, Pelletier SJ, et al. Impact of the model for end-stage liver disease allocation policy on the use of high-risk organs for liver transplantation. Gastroenterology. 2008; 135:15681574. [PubMed: 19009713]

7. Schaubel DE, Sima CS, Goodrich NP, et al. The survival benefit of deceased donor liver transplantation as a function of candidate disease severity and donor quality. Am J Transplant. 2008; 8:419-425. [PubMed: 18190658]

8. Deyo RA, Cherkin DC, Ciol MA. Adapting a clinical comorbidity index for use with ICD-9-CM administrative databases. J Clin Epidemiol. 1992; 45:613-619. [PubMed: 1607900]

9. Longworth L, Bryan S. An empirical comparison of EQ-5D and SF-6D in liver transplant patients. Health Econ. 2003; 12:1061-1067. [PubMed: 14673814]

10. Ware JE Jr, Sherbourne CD. The MOS 36-item short-form health survey (SF-36). I. Conceptual framework and item selection. Med Care. 1992; 30:473-483. [PubMed: 1593914]

11. Volk ML, Reichert HA, Lok AS, et al. Variation in Organ Quality between Liver Transplant Centers. Am J Transplant. 2011; 11:958-964. [PubMed: 21466651]

12. Bryan S, Ratcliffe J, Neuberger JM, et al. Health-related quality of life following liver transplantation. Qual Life Res. 1998; 7:115-120. [PubMed: 9523492]

13. Kosinski M, Zhao SZ, Dedhiya S, et al. Determining minimally important changes in generic and disease-specific health-related quality of life questionnaires in clinical trials of rheumatoid arthritis. Arthritis Rheum. 2000; 43:1478-1487. [PubMed: 10902749] 


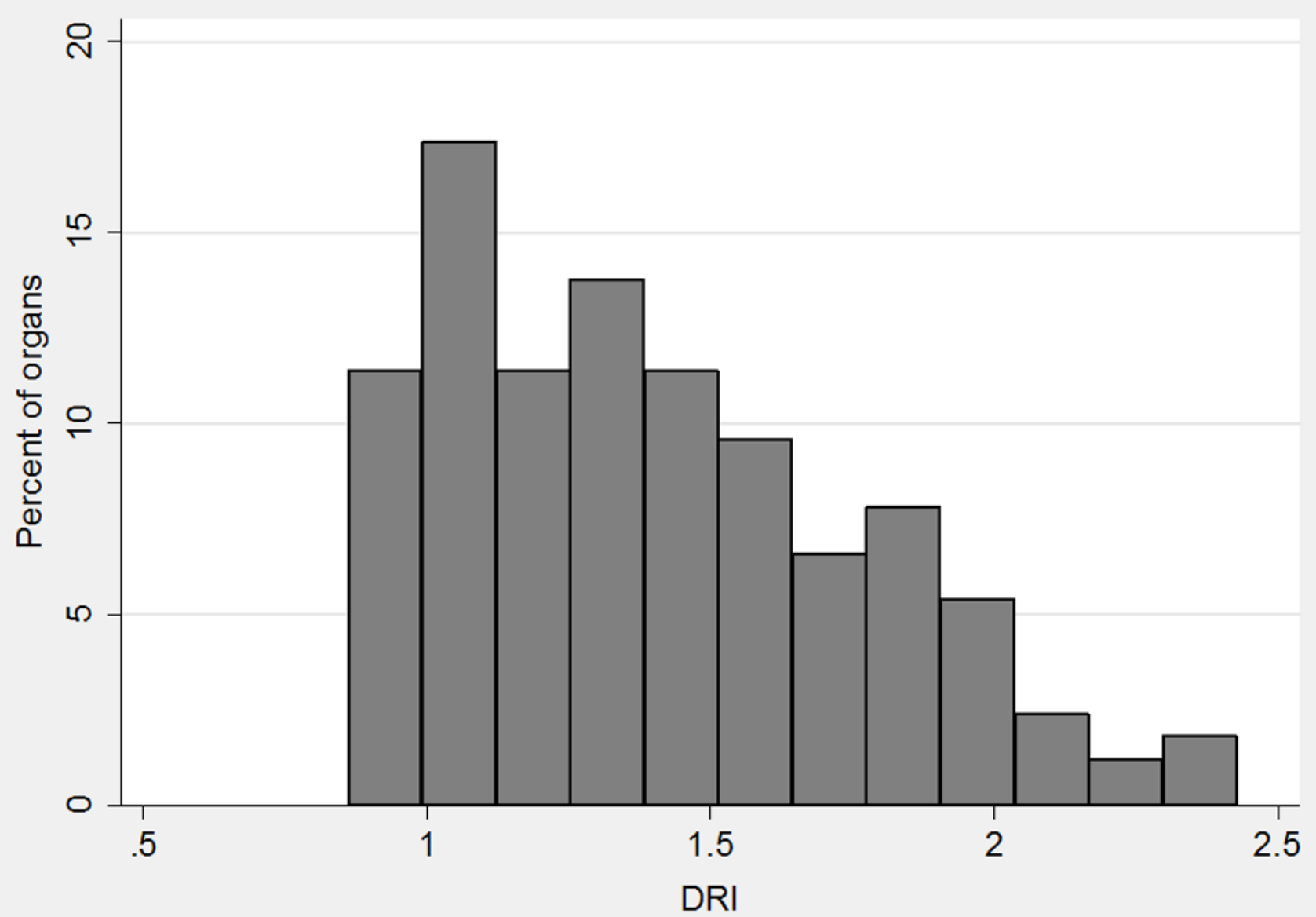

Figure 1.

Distribution of Donor Risk Index (DRI) among organs received by study subjects. 


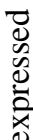

x

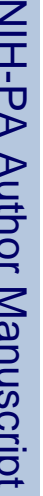

อี

욤

豙

I

昜

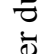

Z

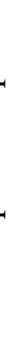

\begin{tabular}{|c|c|c|c|c|c|c|}
\hline 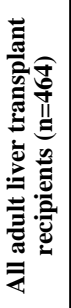 & 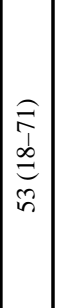 & 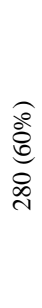 & 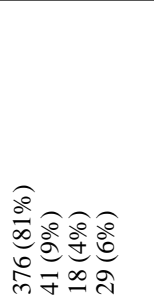 & 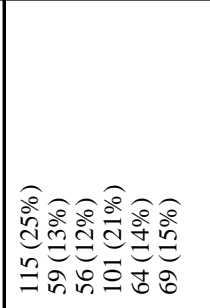 & $\begin{array}{c}0 \\
f \\
0 \\
0 \\
0 \\
-0\end{array}$ & $\frac{\substack{\infty \\
\infty}}{\infty}$ \\
\hline 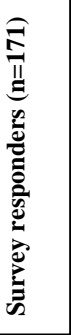 & 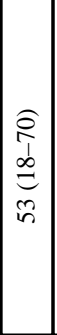 & $\begin{array}{l}\frac{\sigma}{0} \\
\frac{\sigma}{6} \\
\frac{0}{6}\end{array}$ & 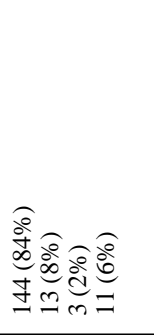 & 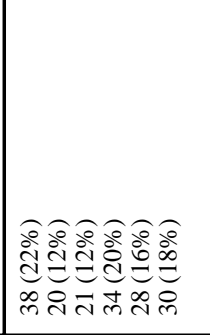 & 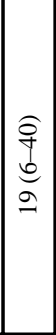 & $\stackrel{\substack{\infty \\
\infty}}{2}$ \\
\hline & 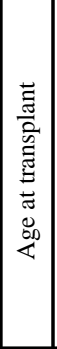 & 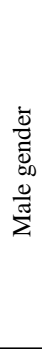 & 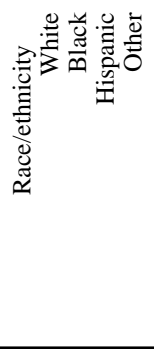 & 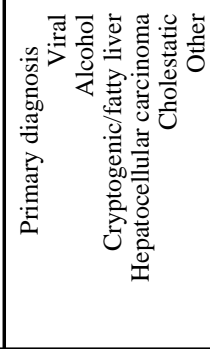 & 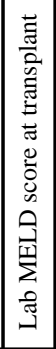 & 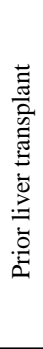 \\
\hline
\end{tabular}

Liver Transpl. Author manuscript; available in PMC 2012 December 1. 


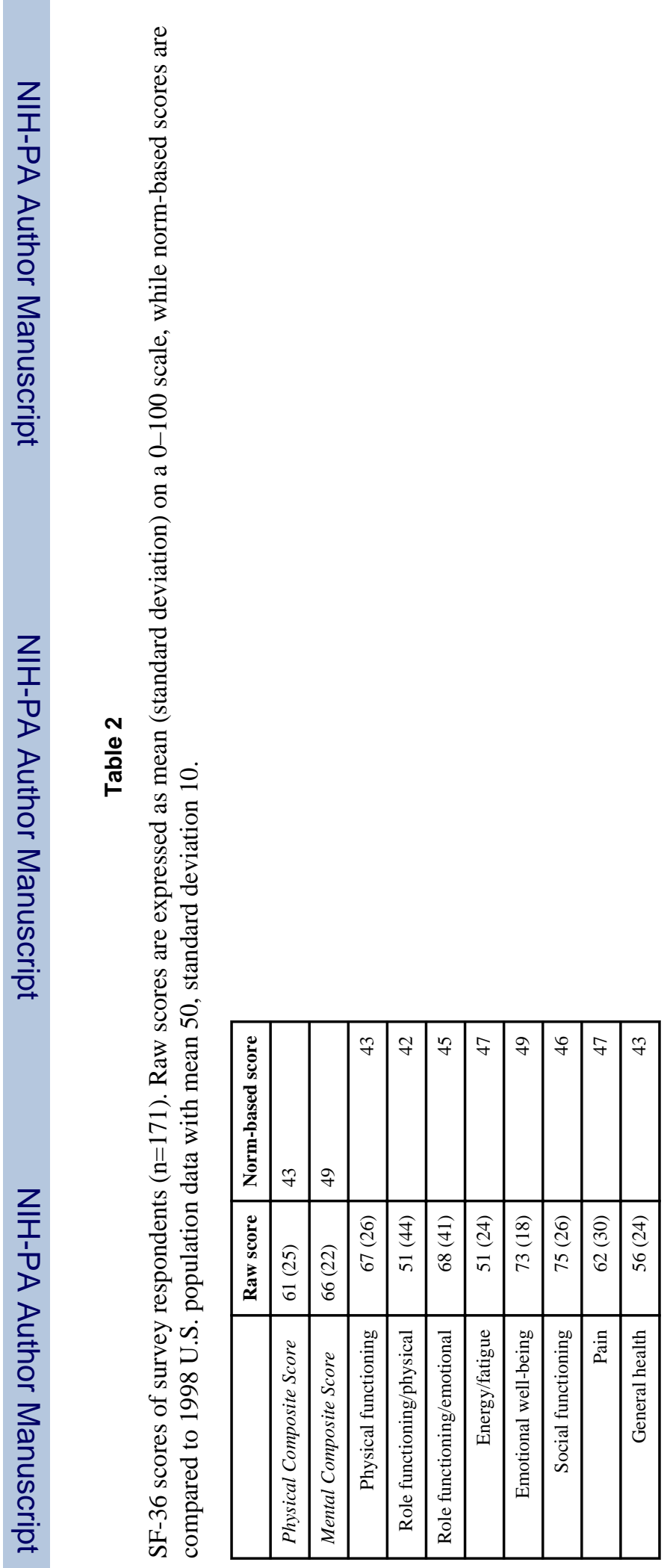

Liver Transpl. Author manuscript; available in PMC 2012 December 1. 
Table 3

Multivariable analysis of pre-transplant factors associated with Physical Composite Score and Mental Composite Score of SF-36. Coefficients should be interpreted as follows: change in SF-36 score per unit increase in DRI.

\begin{tabular}{|l|c|c|c|c|}
\hline & \multicolumn{2}{|c|}{$\begin{array}{c}\text { Physical Composite } \\
\text { Score (PCS) }\end{array}$} & \multicolumn{2}{c|}{$\begin{array}{c}\text { Mental Composite } \\
\text { Score (MCS) }\end{array}$} \\
\hline & Coefficient & $\begin{array}{c}\text { P- } \\
\text { value }\end{array}$ & Coefficient & $\begin{array}{c}\text { P- } \\
\text { value }\end{array}$ \\
\hline DRI & -4.8 & 0.4 & -2.8 & 0.6 \\
\hline Gender & 0.8 & 0.8 & 4.7 & 0.2 \\
\hline Current age & -0.3 & 0.3 & 0.2 & 0.3 \\
\hline MELD at transplant & -0.2 & 0.5 & -.06 & 0.8 \\
\hline Years since transplant & 0.3 & 0.8 & 0.6 & 0.5 \\
\hline Prior transplant & 11.5 & 0.2 & 10.9 & 0.1 \\
\hline Charlson Comorbidity Index & 0.1 & 0.9 & 0.5 & 0.4 \\
\hline Hospitalized at transplant & -2.7 & 0.7 & -4.7 & 0.4 \\
\hline
\end{tabular}


Table 4

Post-transplant complications according to quality of organ received. Data are expressed as median (range) or $\mathrm{n}(\%)$. PTC $=$ Percutaneous transhepatic cholangiogram.

\begin{tabular}{|l|c|c|c|}
\hline & $\begin{array}{c}\text { High risk } \\
\text { (DRI>1.6) organ } \\
\text { recipients (n=52) }\end{array}$ & $\begin{array}{c}\text { Standard risk } \\
\text { organ recipients } \\
(\mathbf{n = 1 1 9})\end{array}$ & P- value \\
\hline Length of stay & $10(5-69)$ & $10(4-101)$ & 0.2 \\
\hline Dialysis beyond transplant hospitalization & $2(4 \%)$ & $14(12 \%)$ & 0.1 \\
\hline Biliary complications requiring PTC & $7(13 \%)$ & $14(12 \%)$ & 0.8 \\
\hline Biopsy-proven rejection & $3(6 \%)$ & $26(22 \%)$ & 0.01 \\
\hline
\end{tabular}

